\title{
A Career in Theoretical Physics
}


Published

Vol. 1 Gauge Theories - Past and Future edited by R. Akhoury, B. de Wit and P. van Nieuwenhuizen

Vol. 2 Scientific Highlights in Memory of Léon van Hove edited by $F$. Nicodemi

Vol. 3 Selected Papers, with Commentary, of T. H. R. Skyrme edited by $G$. E. Brown

Vol. 4 Salamfestschrift edited by A. Ali, J. Ellis and S. Randjbar-Daemi

Vol. 5 Selected Papers of Abdus Salam (with Commentary) edited by A. Ali, C. Isham, T. Kibble and Riazuddin

Forthcoming

Vol. 6 Research on Particle Detectors by G. Charpak 


\section{A CAREER IN THEORETICAL PHYSICS}

\section{P W Anderson}

Department of Physics

Princeton University 
Published by

World Scientific Publishing Co. Pte. Ltd.

P O Box 128, Farrer Road, Singapore 9128

USA office: Suite 1B, 1060 Main Street, River Edge, NJ 07661

UK office: 73 Lynton Mead, Totteridge, London N20 8DH

While every effort has been made to contact the publishers of reprinted papers prior to publication, we have not been successful in a few cases. Where we could not contact the publishers, we have acknowledged the source of the materia. Proper credit will be given to these publishers in future editions of this work after permission is granted.

The author and publisher would like to thank the following publishers of the various journals and books for their assistance and permission to include the selected reprints found in this volume:

Academic Press

Addison-Wesley

American Assoc. Adv. Sci.

American Institute of Physics

American Physical Society

Elsevier Science Publishers

Gordon and Breach Science Publishers

IAEA
IDSET

Marcel Dekker

MIT Press

The Nobel Foundation

Pergamon Press

Plenum Publishing

Società Italiana di Fisica

Taylor and Francis

\section{Library of Congress Cataloging-in-Publication}

Anderson, P. W. (Philip W.), 1923-

A career in theoretical physics / P. W. Anderson.

vol. 7)

p. $\quad \mathrm{cm}$. -- (World Scientific series in 20th century physics ;

ISBN 981021717X. -- ISBN 9810217188 (pbk.)

1. Mathematical physics. I. Title. II. Series.

QC20.A55 1994

$530.1--\mathrm{dc} 20$

94-13919

CIP

Copyright $\odot 1994$ by World Scientific Publishing Co. Pte. Ltd.

All rights reserved. This book, or parts thereof, may not be reproduced in any form or by any means, electronic or mechanical, including photocopying, recording or any information storage and retrieval system now known or to be invented, without written permission from the Publisher.

For photocopying of material in this volume, please pay a copying fee through the Copyright Clearance Center, Inc., 27 Congress Street, Salem, MA 01970, USA. 


\section{INTRODUCTORY ESSAY}

It was in the winter of 1966-7 that I first tried to express my philosophy of what was important in science - or at least, of what was important that was possible for me. I had been invited to spend a month in the pleasant climate of La Jolla as the Regents' lecturer at the UCSD, visiting a department staffed by many of my old friends from Bell Labs, such as Bernd Matthias, Harry Suhl, and George Feher, who had been recruited by Walter Kohn, who was also by courtesy a Bell alumnus. One of the lectures I gave was called "More is Different". The original version, now lost, went over with the audience like a lead balloon, at least according to that portion of the audience most closely related to me. But a cleaned up version, which was published in Science in 1971, has been surprisingly influential. I have chosen to reprint it, out of chronological order, as an additional introduction to this collection.

I already had this overall vision, if only very vaguely, as I completed my thesis and other early work on the breadths of spectral lines (see paper 2 in the book). This work was among the first to approach what was normally thought of as a dissipative process and dealt with by Boltzmann equations for the microscopic variables, in terms of the macroscopic moments of the system as a whole in my own mind called it the "one big molecule" method. I was beginning to see that somehow, starting from the fundamental atomic-level laws of quantum theory, and without any more or less mystical appeals to the difference between the atomic domain and the macroscopic domain where deterministic Newtonian physics held way, one could really understand many of the properties of matter in that macroscopic domain. I also experienced two other insights: that these properties were often extraordinary; and that this understanding was often intellectually challenging and deep, at a level which was for me at least more exciting and worthwhile than any other purely intellectual exercise.

Thus I was launched into the search for the "Aufbauprinzips" of the everyday world. I admired Fröhlich's book on dielectric theory because it took the same point of view; I encountered it in my early work on ferroelectrics (papers 1 and 3). Much more important, I came to see the crucial importance of the concept of broken symmetry, which has been a lifelong interest (papers 6, 7, 9, 10, 11, 15, 22, 26, 32, 39). Broken symmetry is the clearest instance of the process of emergence which lies behind "More is different". My interest began with the antiferromagnetic ground state work in 1952-3, from which I developed the concept of the Goldstone boson. In applying similar thinking to superconductivity, the Higgs phenomenon and the concept of Generalized Rigidity arose, the latter referring to the similarities between superfluidity, superconductivity, and elastic rigidity of all sorts. The topological theory of defects and dissipation, of which papers (7), (11) and 
(27) are examples, is another facet of the conceptual structure of broken symmetry. Other examples were omitted for reasons of space.

The first natural direction in which to seek for understanding of the real world is the explicit quantitative calculation of properties of real materials. This enterprise is particularly rewarding when the basic principles which govern these properties are new, or when the effects are subtle. This has been the mainspring of much of my work, from the early work on magnetic resonance and other spectra, to superexchange (paper 8), the microscopic theory of superconductivity (papers $4,5,9,14,18,30,33$ ), and even work on the electronic structure of solids such as paper 21, foreshadowing the corrections to local density theory, and paper 29 where the idea is to develop a method which explains and exploits the strong locality of the chemical bond, perhaps the most fundamental observation in the field of chemistry, which nonetheless seems to interest quantum chemists not at all.

In 1956 , stimulated by a pioneering paper on percolation by Hammersley and by some puzzling experiments, I started to look at the next level of complexity, those phenomena which are intrinsic to disorder. The first result was the theory of localisation of waves (papers 25, 28, 16). This was slow of acceptance, and, perhaps discouraged by this fact, it was not until 1969 that I returned to it and also began to look at other disorder phenomena such as glass and spin glass (papers 23, 24, 34). Spin glass stimulated a very fertile period of worldwide activity, first in the development of totally novel methodology, such as a new statistical mechanis of nonergodic systems, and then in applications of the new methodology to a "cornucopia" of new ideas and concepts and problems: neural networks, computer algorithms, rugged evolutionary landscapes, even immunology. This has left me involved in such fields as economics and the origin of life. The interest in complex, emergent phenomena made it natural for me to participate at an early stage in the Santa Fe Institute, founded on the twin principles of interdisciplinarity and of the importance and tractability of genuinely complex systems. There has not been much space for these ideas; only papers $24,31,34$ and 39 touch on them.

Left behind, in my old field of quantum condensed matter physics, was a worry about the validity and completeness of the theories of many-body quantum systems which had occupied that world in the 1950's and 60's. My work on the Anderson model of magnetic impurities (paper 12) had led on to the "infrared catastrophe" in Fermi systems which was the key to my work on the Kondo phenomenon (papers 13, 20) which solved that isolated many-body system but only at the cost of going beyond the conventional perturbation theory. I was also disturbed by a class of strongly interacting superconducting materials (30), as well as our notable lack of success in dealing with the field of quantum valence fluctuations, i.e. "heavy electrons" $(35,36,37)$. With the appearance of the high $T_{\mathrm{c}}$ cuprates in 1987, my apprehensions became manifestly justified, and I began to formulate theories of metals based on new principles (papers 40, 41, 42, 43). This is the area which now most absorbs me, and where my efforts have been rewarded with the discovery of 
several new and fascinating phenomena. I reach my 70th year still involved in two major, very different types of problem. Although there is no easy way to round this one off, I have tried to give a flavor of the ideas involved.

My original vision seems to me to have been sound. The problem of understanding the "here and now" world seems to show no sign of being exhausted. The contribution of physics is the method of dealing correctly both with the substrate from which emergence takes place, and with the emergent phenomenon itself. Examples are legion: for one, superconductivity cannot be properly understood simply as a phenomenology without understanding electrons and their interactions, nor on the other hand as a property of a small number of electrons without taking into account the macroscopic system. Ever newer insights into the nature of the world around us will continually arise from this style of doing science. There will be physics even in a world without the SSC.

Regarding the choice of articles in this book: the sheer volume of either a complete selection of research articles or, even more, a complete set of reviews would have been much too great. I have given preference to materials which are for one reason or another not easy of access: lecture notes and out of print sources, for example. I have tried but failed to provide a selection of all major topics; the book is long enough. I have included a few items on history as I saw it, and on general aspects of science.

Oxford, December 2, 1993 
This page is intentionally left blank 


\section{CONTENTS}

Introductory Essay $\quad$ v

More is Different $\quad 1$

1. Theory of Ferroelectric Behaviour of Barium Titanate $\quad 5$

2. Use of Stochastic Methods in Line-Broadening Problems $\quad 13$

3. Qualitative Considerations on the Statistics of the Phase Transition in $\mathrm{BaTiO}_{3}$-type Ferroelectrics 35

4. Theory of Dirty Superconductors 45

5. Calculation of the Superconducting State Parameters with Retarded Electron-Phonon Interaction $\quad 51$ (with P. Morel)

6. Plasmons, Gauge Invariance, and Mass 61

$\begin{array}{lr}\text { 7. Hard Superconductors } & 67\end{array}$ Hard Superconductivity: Theory of the Motion of Abrikosov Flux Lines 107 (with Y. B. Kim)

8. Exchange in Magnetic Insulators 113

$\begin{array}{ll}\text { 9. Superconductivity } & 131\end{array}$ (with B. T. Matthias)

10. Coherent Matter Field Phenomena in Superfluids 143

11. Considerations on the Flow of Superfluid Helium 165

12. Multiple-Scattering Theory and Resonances in Transition Metals 179 (with W. L. McMillan)

13. The Kondo Effect I $\quad 217$

The Kondo Effect II $\quad 224$

Kondo Effect III: The Wilderness - Mainly Theoretical 232

Kondo Effect IV: Out of the Wilderness 238

14. Superconductivity in the Past and Future 245 
15. Macroscopic Coherence and Superfluidity

16. The Fermi Glass: Theory and Experiment 273

17. Space-Time and Scaling Techniques in the Kondo Problem 281

18. Comments on the Maximum Superconducting Transition Temperature 287

Comment on 'Model for an Exciton Mechanism of Superconductivity' 299 (with J. C. Inkson)

19. Conference Summary 303

20. Asymptotically Exact Methods in the Kondo Problem 311 (with G. Yuval)

21. Many-Body Effects at Surfaces 333

22. Uses of Solid State Analogies in Elementary Particle Theory 363

23. Possible Consequences of Negative U Centers in Amorphous Materials 389

24. Survey of Theories of Spin Glass 395

25. Disorder: A Frontier of Theoretical Physics 413

26. Some General Thoughts about Broken Symmetry 419

27. The Rheology of Neutron Stars: Vortex Line Pinning in the Crust Superfluid

(with M. A. Alpar, D. Pines and J. Shaham)

28. Localization Redux

29. Chemical Pseudopotentials 453

30. Some Remarks on Strong Electron-Phonon Coupling Metals 463 (with C. C. Yu)

31. Spin Glass Hamiltonians: A Bridge between Biology, Statistical Mechanics and Computer Science $\quad 495$

32. Measurement in Quantum Theory and the Problem of Complex Systems 501

33. It's Not Over Till the Fat Lady Sings 515

34. Spin Glass I: A Scaling Law Rescued 525

Spin Glass II: Is There a Phase Transition? 528

Spin Glass III: Theory Raises its Head $\quad 529$ 
Spin Glass IV: Glimmerings of Trouble

Spin Glass V: Real Power Brought to Bear 533

Spin Glass VI: Spin Glass as Cornucopia $\quad 535$

Spin Glass VII: Spin Glass as Paradigm $\quad 537$

35. Valence Instabilities and Related Narrow-Band Phenomena 539

36. Present Status of Theory: 1/N Approach 548

37. The Problem of Fluctuating Valence in f-Electron Metals 562

38. Some Ideas on the Aesthetics of Science 569

39. Theoretical Paradigms for the Sciences of Complexity 584

40. 50 Years of the Mott Phenomenon: Insulators, Magnets, Solids, and Superconductors as Aspects of Strong-Repulsion Theory $\quad 595$

41. The "Central Dogmas" 637

42. The "Infrared Catastrophe": When Does it Trash Fermi Liquid Theory? 657

43. Experimental Constraints on the Theory of High- $T_{c}$ Superconductivity 673 
This page is intentionally left blank 


\section{CONTENTS}

(in Sections)

Introductory Essay

More is Different

Ferroelectricity, Soft Modes

[1] Theory of Ferroelectric Behaviour of Barium Titanate

[3] Qualitative Considerations on the Statistics of the Phase Transition in $\mathrm{BaTiO}_{3}$-type Ferroelectrics

\section{Line Broadening, Correlation Function}

[2] Use of Stochastic Methods in Line-Broadening Problems

Superconductivity: BCS

[4] Theory of Dirty Superconductors

[5] Calculation of the Superconducting State Parameters with Retarded Electron-Phonon Interaction

[7] Hard Superconductors 67

Hard Superconductivity: Theory of the Motion of Abrikosov Flux Lines 107

[9] Superconductivity 131

[14] Superconductivity in the Past and Future 245

[15] Macroscopic Coherence and Superfluidity 263

[18] Comments on the Maximum Superconducting Transition Temperature 287

Comment on 'Model for an Exciton Mechanism of Superconductivity' 299

[30] Some Remarks on Strong Electron-Phonon Coupling Metals 463

Broken Symmetry

[6] Plasmons, Gauge Invariance, and Mass 61

[10] Coherent Matter Field Phenomena in Superfluids 143 
[11] Considerations on the Flow of Superfluid Helium

[22] Uses of Solid State Analogies in Elementary Particle Theory 363

[26] Some General Thoughts about Broken Symmetry

Magnetic State, Mott Insulators

[8] Exchange in Magnetic Insulators

Superfluidity: $\mathrm{He}^{3}+\mathrm{He}^{4}$

[11] Considerations on the Flow of Superfluid Helium 165

[15] Macroscopic Coherence and Superfluidity 263

[27] The Rheology of Neutron Stars: Vortex Line Pinning in the Crust Superfluid

Local Moments and Kondo Effect

[12] Multiple-Scattering Theory and Resonances in Transition Metals 179

[13] The Kondo Effect I 217

The Kondo Effect II 224

Kondo Effect III: The Wilderness - Mainly Theoretical 232

Kondo Effect IV: Out of the Wilderness 238

[17] Space-Time and Scaling Techniques in the Kondo Problem 281

[20] Asymptotically Exact Methods in the Kondo Problem 311

Localization

[16] The Fermi Glass: Theory and Experiment 273

[23] Possible Consequences of Negative U Centers in Amorphous Materials 389

[25] Disorder: A Frontier of Theoretical Physics 413

[28] Localization Redux 445

\section{General}

[19] Conference Summary

[27] The Rheology of Neutron Stars: Vortex Line Pinning in the

Crust Superfluid 
[31] Spin Glass Hamiltonians: A Bridge between Biology, Statistical

Mechanics and Computer Science

[32] Measurement in Quantum Theory and the Problem of Complex Systems

[33] It's Not Over Till the Fat Lady Sings

[38] Some Ideas on the Aesthetics of Science

[39] Theoretical Paradigms for the Sciences of Complexity

\section{Electronic Structure}

[12] Multiple-Scattering Theory and Resonances in Transition Metals 179

[21] Many-Body Effects at Surfaces 333

[29] Chemical Pseudopotentials 453

\section{Spin Glass and Non-Ergodic Systems}

[23] Possible Consequences of Negative U Centers in Amorphous Materials 389

[24] Survey of Theories of Spin Glass 395

[34] Spin Glass I: A Scaling Law Rescued 525

Spin Glass II: Is There a Phase Transition? 528

Spin Glass III: Theory Raises its Head 529

Spin Glass IV: Glimmerings of Trouble 531

Spin Glass V: Real Power Brought to Bear 533

Spin Glass VI: Spin Glass as Cornucopia

Spin Glass VII: Spin Glass as Paradigm 537

\section{Mixed Valence}

[35] Valence Instabilities and Related Narrow-Band Phenomena 539

[36] Present Status of Theory: 1/N Approach 548

[37] The Problem of Fluctuating Valence in f-Electron Metals 562

High- $T_{c}$ and the New Physics

[40] 50 Years of the Mott Phenomenon: Insulators, Magnets, Solids, and Superconductors as Aspects of Strong-Repulsion Theory 
[41] The "Central Dogmas" 637

[42] The "Infrared Catastrophe": When Does it Trash Fermi Liquid Theory? 657

[43] Experimental Constraints on the Theory of High- $T_{c}$ Superconductivity 673 